\title{
Closed-form integration of singular terms for constant, linear and quadratic boundary elements. Part 2. SV-P wave propagation
}

\author{
A.J.B. Tadeu ${ }^{\mathrm{a}, *}$, P.F.A. Santos ${ }^{\mathrm{a}}$, E. Kausel ${ }^{\mathrm{b}}$ \\ ${ }^{a}$ Department of Civil Engineering, University of Coimbra, 3020 Coimbra, Portugal \\ ${ }^{\mathrm{b}}$ Department of Civil and Environmental Engineering, Massachusetts Institute of Technology, Cambridge, MA 02139, USA
}

Received 17 November 1998; accepted 11 May 1999

\begin{abstract}
Part I of these two related papers considered the analytical evaluation of singular integrals for anti-plane boundary elements, the results of which were then applied to the evaluation of scattering problems involving SH waves. This second part provides an extension of these results to the more complicated case of in-plane boundary elements, and presents their application to scattering problems involving SV-P waves. First, the singular integrals for constant, linear and quadratic boundary elements are evaluated a in closed form. Thereafter, the formulation is used to model cylindrical inclusions in a two-dimensional elastic medium illuminated by dynamic in-plane (plane-strain) line sources. The boundary element method (BEM) results are then compared with the known analytical solutions for these problems. (C) 1999 Elsevier Science Ltd. All rights reserved.
\end{abstract}

Keywords: Wave propagation; Shear waves; Elastic inclusions; Scattering; Boundary element method; Singular integrals

\section{Introduction}

Part I of these two related papers [1] considered the analytical evaluation of singular integrals for anti-plane boundary elements and their application to scattering problems with SH waves. This second part extends those results to the more complicated case of in-plane (plane strain) boundary elements, and applies them to scattering problems involving SV-P waves. First, the singular integrals for constant, linear and quadratic boundary elements are evaluated analytically. These exact integrals are then compared with those obtained with Gaussian quadrature, which both demonstrates the validity of the analytical expressions presented and also allows us to assess the accuracy of the numerical method. Thereafter, the formulation is used to model cylindrical inclusions in a two-dimensional elastic medium insonified (or illuminated) by dynamic in-plane (i.e. plane-strain) line sources. Finally, the boundary element method (BEM) results are compared with the known closed-form solutions for these scattering problems.

\section{Boundary element formulation}

The boundary element formulation for in-plane wave

\footnotetext{
* Corresponding author.
}

motion involves the integrals [2-4]

$$
\begin{aligned}
& H_{i j}^{k l}=\int_{C_{l}} \phi H_{i j}\left(x_{k}, x_{l}, n_{l}\right) \mathrm{d} C_{l} \quad(i, j=1,2) \\
& G_{i j}^{k l}=\int_{C_{l}} \phi G_{i j}\left(x_{k}, x_{l}\right) \mathrm{d} C_{l} \quad(i, j=1,2)
\end{aligned}
$$

in which $G_{i j}\left(x_{k}, x_{l}\right)$ and $H_{i j}\left(x_{k}, x_{l}, n_{l}\right)$ are, respectively, the components of the Green's tensor for displacement and traction components at the observation point $x_{k}$ in direction $i$ caused by a concentrated load acting at the source point $x_{l}$ in direction $j$. Also, $n_{l}$ is the unit outward normal for the $l$ th boundary segment $C_{l}$, and $\phi$ contains the interpolation functions. The requisite Green's function for this problem is [5]

$$
\begin{aligned}
G_{i j}\left(x, x_{0}\right)= & i /(4 \mu)\left\{-\delta_{i j} H_{0}\left(k_{\beta} r\right)+\delta_{i j} /\left(k_{\beta} r\right)\left\lfloor H_{1}\left(k_{\beta} r\right)\right.\right. \\
& \left.\left.-\xi H_{1}\left(k_{\alpha} r\right)\right\rfloor\right\}-i /(4 \mu) \\
& \times\left\{\frac{\partial r}{\partial x_{i}} \frac{\partial r}{\partial x_{j}}\left\lfloor H_{2}\left(k_{\beta} r\right)-\xi^{2} H_{2}\left(k_{\alpha} r\right)\right\rfloor\right\}
\end{aligned}
$$

$(i, j=1,2)$.

In this equation $\alpha=\sqrt{(\lambda+2 \mu) / \rho}$ and $\beta=\sqrt{\mu / \rho}$ are the velocities of dilatational and shear waves, respectively, $\lambda$ and $\mu$ are the Lamé constants, $k_{\alpha}=\omega / \alpha$ and $k_{\beta}=\omega / \beta$ are the wave-numbers, $\omega$ the circular frequency; $\rho$ the mass 

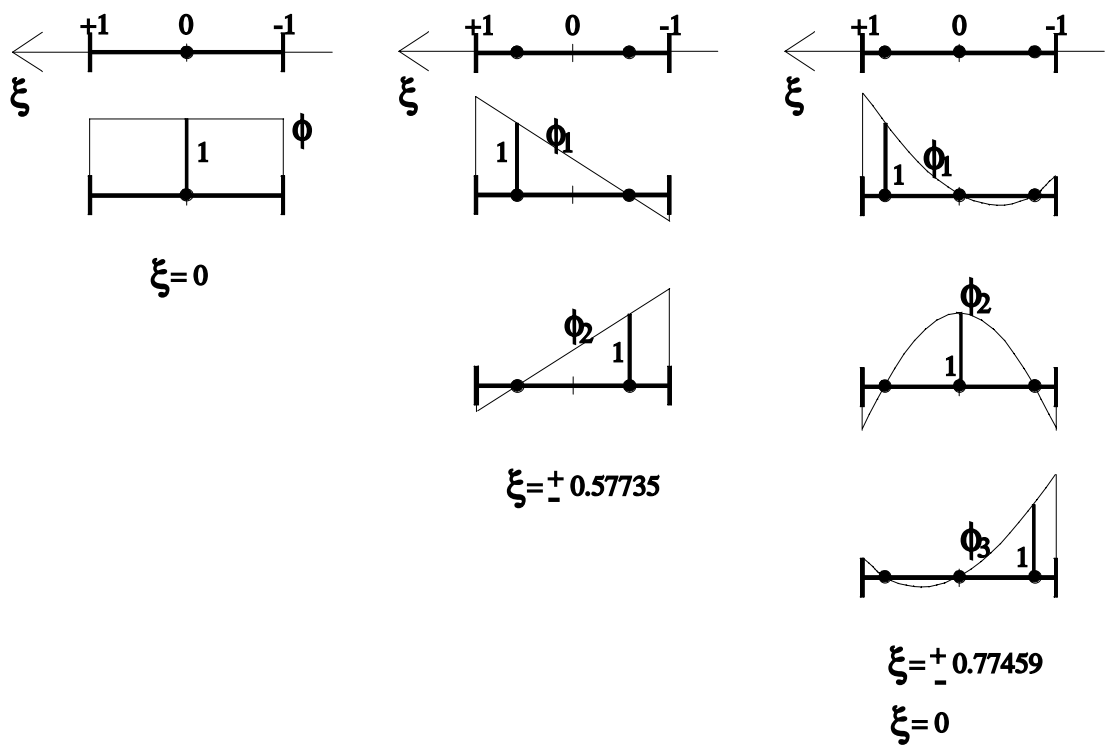

Fig. 1. Interpolation functions and position of the Gauss-Legendre nodes.

density, $r=\left|x-x_{0}\right|$ the source-receiver distance, $H_{n}()=$ $H_{n}^{(2)}()$ the Hankel function of the second kind of order $n, \delta_{i j}$ the Kronecker delta, and $i=\sqrt{-1}$ (not to be confused with the sub-index $i$ indicating direction). The corresponding expressions for the tractions $H_{i j}$, which may be obtained from $G_{i j}$ by taking partial derivatives, are omitted here for the sake of brevity.

\section{Element integration}

As in the anti-plane formulation, discontinuous elements are used here to allow discontinuity of stresses between contiguous elements. The interpolation functions used are depicted in Fig. 1, which shows the elements' nodes coinciding with the sampling points in the Gauss-Legendre numerical integration.

When the element to be integrated is not the loaded element in Eqs. (1) and (2), the integrands are non-singular and the integration is best carried out using the standard Gaussian quadrature. For the loaded element, however, the integrands exhibit a singularity, but it is then possible to carry out the integration in a closed form, as described

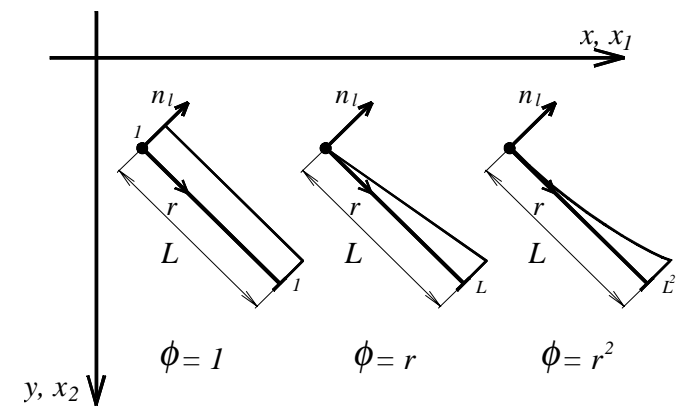

Fig. 2. The local co-ordinate system for segment integration. below. To demonstrate this assertion, consider the $l$ th singular segment of length $L$ shown in Fig. 2, together with constant, linear and quadratic interpolation functions $\phi$.

The integration of the Green's functions can be expressed in terms of the following integrals:

$$
\begin{aligned}
& \int G_{i j} \mathrm{~d} r ; \quad \int r G_{i j} \mathrm{~d} r ; \quad \int r^{2} G_{i j} \mathrm{~d} r ; \quad \int H_{i j} \mathrm{~d} r ; \\
& \int r H_{i j} \mathrm{~d} r ; \quad \int r^{2} H_{i j} \mathrm{~d} r \quad(i, j=1,2) .
\end{aligned}
$$

The $H_{i j}$ are obtained from the derivatives of the $G_{i j}$, namely,

$$
\begin{aligned}
\frac{\partial G_{i j}}{\partial x_{k}}= & \frac{i}{4 \mu}\left[\left\{\delta_{i j}\left(C_{1}+C_{2}+C_{3}\right)-\left(\frac{\partial r}{\partial x_{i}}\right)\left(\frac{\partial r}{\partial x_{j}}\right) C_{4}\right.\right. \\
& \left.+2\left(\frac{\partial r}{\partial x_{i}}\right)\left(\frac{\partial r}{\partial x_{j}}\right) C_{5}\right\} \frac{\partial r}{\partial x_{k}} \\
& \left.-\left(\delta_{i k} \frac{\partial r}{\partial x_{j}}+\delta_{j k} \frac{\partial r}{\partial x_{i}}\right) C_{5}\right] \quad(i, j, k=1,2)
\end{aligned}
$$

where

$$
\begin{aligned}
& C_{1}=C_{1}(r)=k_{\beta} H_{1}\left(k_{\beta} r\right) \\
& C_{2}=C_{2}(r)=-1 /\left(k_{\beta} r^{2}\right)\left\{H_{1}\left(k_{\beta} r\right)-\xi H_{1}\left(k_{\alpha} r\right)\right\} \\
& C_{3}=C_{3}(r)=1 / k_{\beta} r\left\{-H_{1}\left(k_{\beta} r / r+k_{\beta} H_{0}\left(k_{\beta} r\right)\right.\right. \\
& \left.-\xi\left(-H_{1}\left(k_{\alpha} r\right) / r+k_{\alpha} H_{0}\left(k_{\alpha} r\right)\right)\right\}
\end{aligned}
$$




$$
\begin{aligned}
C_{4}=C_{4}(r)= & \left\{-2 / r H_{2}\left(k_{\beta} r\right)+k_{\beta} H_{1}\left(k_{\beta} r\right)\right. \\
& \left.-\xi^{2}\left(-2 / r H_{2}\left(k_{\alpha} r\right)+k_{\alpha} H_{1}\left(k_{\alpha} r\right)\right)\right\} \\
C_{5}=C_{5}(r)= & \left\{H_{2}\left(k_{\beta} r\right) / r-\xi^{2} H_{2}\left(k_{\alpha} r\right) / r\right\} .
\end{aligned}
$$

The partial derivatives $\partial r / \partial x_{i}, \partial r / \partial x_{j}$, in Eq. (5) represent the slope of the line connecting the nodal point with the integration point. As for the loaded element this equals the slope of the segment, which is constant, it follows that these derivatives do not affect the integration of the singular terms. To evaluate the various integrals, it is necessary to make use of the recurrence relations for the Bessel functions, the ascending series for these functions and the integrals listed in Appendix A [6]. Still, a considerable algebra is necessary, as discussed later.

\subsection{Integration of $\int_{0}^{L} G_{i j} \mathrm{~d} r$}

In view of Eq. (3), this integral may be written as

$G_{i j}=\int_{0}^{L} G_{i j}\left(x, x_{r}\right) \mathrm{d} r=\frac{i}{4 \mu}\left[\delta_{i j}\left(-B_{1}+B_{2}\right)-\frac{\partial r}{\partial x_{i}} \frac{\partial r}{\partial x_{j}} B_{3}\right]$

$i, j=1,2$

with

$B_{1}=\int_{0}^{L} H_{0}\left(k_{\beta}^{r}\right) \mathrm{d} r$

$B_{2}=\int_{0}^{L} \frac{1}{k_{\beta} r}\left\{H_{1}\left(k_{\beta} r\right)-\xi H_{1}\left(k_{\alpha} r\right) \mathrm{d} r\right.$

$B_{3}=\int_{0}^{L}\left\{H_{2}\left(k_{\beta} r\right)-\xi^{2} H_{2}\left(k_{\alpha} r\right) \mathrm{d} r\right.$.

The first integral, namely $B_{1}$, was obtained in Part 1 ,

$$
\begin{aligned}
B_{1} & =B_{1}(b)=\int_{0}^{L} H_{0}\left(k_{\beta} r\right) \mathrm{d} r=\int_{0}^{L}\left[J_{0}\left(k_{\beta} r\right)-i Y_{0}\left(k_{\beta} r\right)\right] \mathrm{d} r \\
& =L\left[I_{1}(b)-i I_{2}(b)\right]
\end{aligned}
$$

with $b=k_{\beta} L$ and

$$
\begin{aligned}
& I_{1}(b)=J_{0}(b)+\frac{\pi}{2}\left[S_{0}(b) J_{1}(b)-S_{1}(b) J_{0}(b)\right] \\
& I_{2}(b)=Y_{0}(b)+\frac{\pi}{2}\left[S_{0}(b) Y_{1}(b)-S_{1}(b) Y_{0}(b)\right]
\end{aligned}
$$

where $S_{0}()$ and $S_{1}\left(\right.$ ) are Struve functions and $J_{n}()$ and $Y_{n}()$ are $n$th order Bessel functions of the first and second kind, respectively.

To evaluate $B_{2}$ and $B_{3}$, it is necessary to make use of the recurrence relations for the Bessel functions, Eq. (A1), and to dispose of an apparent singularity at the lower limit of the integrals. This may be accomplished by considering the ascending series for the Bessel functions, Eq. (A6), and observing that the singular terms vanish exactly on account of the fact that $\xi=\beta / \alpha=k_{\beta} / k_{\alpha}$. For example,

$$
\begin{aligned}
B_{2}= & \operatorname{Lim}_{\varepsilon \rightarrow 0} \int_{\varepsilon}^{L}\left\{\left[H_{0}\left(k_{\beta} r\right)-\frac{1}{k_{\beta}} \frac{\mathrm{d} H_{1}\left(k_{\beta} r\right)}{\mathrm{d} r}\right]\right. \\
& \left.-\xi^{2}\left[H_{0}\left(k_{\alpha} r\right)-\frac{1}{k_{\alpha}} \frac{\mathrm{d} H_{1}\left(k_{\alpha} r\right)}{\mathrm{d} r}\right]\right\} \mathrm{d} r \\
= & \int_{0}^{L}\left[H_{0}\left(k_{\alpha} r\right)-\xi^{2} H_{0}\left(k_{\alpha} r\right)\right] \mathrm{d} r \\
& -\frac{1}{k_{\beta}} \operatorname{Lim}_{\varepsilon \rightarrow 0} \int_{\varepsilon}^{L}\left[\frac{\mathrm{d} H_{1}\left(k_{\beta} r\right)}{\mathrm{d} r}-\xi \frac{\mathrm{d} H_{1}\left(k_{\alpha} r\right)}{\mathrm{d} r}\right] \mathrm{d} r \\
= & B_{1}\left(k_{\beta} L\right)-\xi^{2} B_{1}\left(k_{\alpha} L\right)-\frac{1}{k_{\beta}} \underset{\varepsilon \rightarrow 0}{\operatorname{Lim}}\left[H_{1}\left(k_{\beta} r\right)-\xi H_{1}\left(k_{\alpha} r\right)\right]_{\varepsilon}^{L}
\end{aligned}
$$

$B_{2}=B_{1}(b)-\xi^{2} B_{1}(a)-\frac{1}{k_{\beta}}\left[H_{1}(b)-\xi H_{1}(a)\right]$

with $a=k_{\alpha} L$. By similar manipulations with the second recurrence relation for the Bessel function, Eq. (A2), we obtain

$B_{3}=B_{2}-\frac{1}{k_{\beta}}\left[H_{1}(b)-\xi H_{1}(a)\right]$

\subsection{Integration of $\int_{0}^{L} r G_{i j} \mathrm{~d} r$}

In view of Eq. (3), this integral may be written as

$\int_{0}^{L} r G_{i j} \mathrm{~d} r=\frac{i}{4 \mu}\left[\delta_{i j}\left(-B_{1 L}(b)+B_{2 L}\right)-\frac{\partial r}{\partial x_{i}} \frac{\partial r}{\partial x_{j}} B_{3 L}\right]$

$i, j=1,2$

with

$B_{1 L}(b)=\int_{0}^{L} r H_{0}\left(k_{\beta} r\right) \mathrm{d} r$

$B_{2 L}=\int_{0}^{L} \frac{1}{k_{\beta}}\left\{H_{1}\left(k_{\beta} r\right)-\xi H_{1}\left(k_{\alpha} r\right)\right\} \mathrm{d} r$

$B_{3 L}=\int_{0}^{L} r\left\{H_{2}\left(k_{\beta} r\right)-\xi^{2} H_{2}\left(k_{\alpha} r\right)\right\} \mathrm{d} r$.

The first of these integrals was already obtained in Part 1 [1],

$$
\begin{aligned}
B_{1 L}(b) & =\int_{0}^{L} r H_{0}\left(k_{\beta} r\right) \mathrm{d} r \\
& =\left[\frac{L}{k_{\beta}} J_{1}(b)\right]-i\left[\frac{L}{k_{\beta}} Y_{1}(b)+\frac{2}{\pi k_{\beta}^{2}}\right] .
\end{aligned}
$$

To evaluate $B_{2 L}$, it is again necessary to dispose of the 


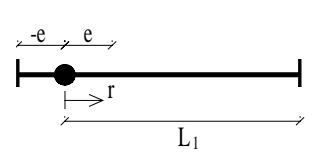

$$
\int_{-e}^{L_{l}} H_{i j} d r=\int_{e}^{L_{l}} H_{i j} d r
$$

Fig. 3. The constant-element integration.

apparent singularity at the lower limit of the integrals. This can be accomplished by considering the ascending series for the Bessel function in Eq. (A8), and verifying that the terms attributable to the singularity vanish exactly because $\xi=$ $\beta / \alpha=k_{\alpha} / k_{\beta}$. For example,

$$
\begin{aligned}
B_{2 L}= & \int_{0}^{L} \frac{1}{k_{\beta}}\left\{H_{1}\left(k_{\beta} r\right)-\xi H_{1}\left(k_{\alpha} r\right)\right\} \mathrm{d} r \\
= & \frac{1}{k_{\beta}}\left[\left(\frac{1}{k_{\beta}}\left(1-J_{0}(b)\right)-\frac{1}{k_{\alpha}} \xi\left(1-J_{0}(a)\right)\right)\right. \\
& \left.-i \operatorname{Lim}_{\varepsilon \rightarrow 0}\left[-\frac{Y_{0}\left(k_{\beta} r\right)}{k_{\beta}}+\xi \frac{Y_{0}\left(k_{\alpha} r\right)}{k_{\alpha}}\right]_{\varepsilon}^{L}\right] \\
= & \frac{1}{k_{\beta}^{2}}\left[\left\{-J_{0}(b)+J_{0}(a)\right\}-i\left\{-Y_{0}(b)+Y_{0}(a)\right.\right. \\
& \left.\left.-\frac{2}{\pi} \ln \xi\right\}\right] .
\end{aligned}
$$

By similar manipulations, and using the recurrence relation for the Hankel function, Eq. (A3), one obtains

$$
\begin{aligned}
B_{3 L} & =\int_{0}^{L}\left\{r H_{2}\left(k_{\beta} r\right)-\xi^{2} r H_{2}\left(k_{\alpha} r\right)\right\} \mathrm{d} r \\
& =2 B_{2 L}-B_{1 L}(b)+\xi^{2} B_{1 L}(a)
\end{aligned}
$$

with

$B_{1 L}(a)=\left[\frac{L}{k_{\alpha}} J_{1}(a)\right]-i\left[\frac{L}{k_{\alpha}} Y_{1}(a)+\frac{2}{\pi k_{\alpha}^{2}}\right]$.

\subsection{Integration of $\int_{0}^{L} r^{2} G_{i j} \mathrm{~d} r$}

In view of Eq. (3), this integral is

$$
\begin{aligned}
& \int_{0}^{L} r^{2} G_{i j} \mathrm{~d} r=\frac{i}{4 \mu}\left[\delta_{i j} C_{1 Q}-\frac{\partial r}{\partial x_{i}} \frac{\partial r}{\partial x_{j}}\left(C_{2 Q}(b)-\xi^{2} C_{2 Q}(a)\right)\right] \\
& i, j=1,2
\end{aligned}
$$

with

$$
C_{1 Q}=-\int_{0}^{L} r^{2} H_{0}\left(k_{\beta} r\right) \mathrm{d} r+\int_{0}^{L} \frac{r}{k_{\beta}}\left\{H_{1}\left(k_{\beta} r\right)-\xi H_{1}\left(k_{\alpha} r\right) \mathrm{d} r\right.
$$

$C_{2 Q}(b)=\int_{0}^{L} r^{2} H_{2}\left(k_{\beta} r\right) \mathrm{d} r$

$C_{2 Q}(a)=\int_{0}^{L} r^{2} H_{2}\left(k_{\alpha} r\right) \mathrm{d} r$.

The evaluation of $C_{2 Q}(a)$ and $C_{2 Q}(b)$ involves the use of the recurrence relation for the Hankel function, Eq. (A2), and an integration by parts,

$$
\begin{aligned}
\int_{0}^{L} r^{2} H_{2}(k r) \mathrm{d} r=\frac{1}{k} \int_{0}^{L} r H_{1}(k r) \mathrm{d} r-\frac{1}{k} \int_{0}^{L} r^{2} \frac{\mathrm{d} H_{1}(k r)}{\mathrm{d} r} \mathrm{~d} r \\
=\frac{3}{k}\left[\operatorname{Lim}_{\varepsilon \rightarrow 0}\left[-\frac{r}{k} H_{0}(k r)\right]_{\varepsilon}^{L}+\frac{1}{k} \int_{0}^{L} H_{0}(k r) \mathrm{d} r\right] \\
\quad-\operatorname{Lim}_{\varepsilon \rightarrow 0}\left[\frac{r^{2}}{k} H_{1}(k r)\right]_{\varepsilon}^{L} .
\end{aligned}
$$

To again dispose of the apparent singularity at the lower limit of the integrals, it suffices to consider the ascending series for the Bessel functions, Eqs. (A5) and (A8), and make use of the known limit for the expression $\operatorname{Lim}_{\varepsilon \rightarrow 0}[(k r) \ln (k r)]_{\varepsilon}=0$. The result is

$$
\begin{aligned}
\int_{0}^{L} r^{2} H_{2}(k r) \mathrm{d} r= & -\frac{3 L}{k^{2}} H_{0}(k L)+\frac{3}{k^{2}} \int_{0}^{L} H_{0}(k r) \mathrm{d} r \\
& -\frac{L^{2}}{k} H_{1}(k L) .
\end{aligned}
$$

Thus,

$$
\begin{aligned}
C_{2 Q}(a) & =\int_{0}^{L} r^{2} H_{2}\left(k_{\alpha} r\right) \mathrm{d} r \\
& =-\frac{3 L}{k_{\alpha}^{2}} H_{0}(a)+\frac{3}{k_{\alpha}^{2}} B_{1}(a)-\frac{L^{2}}{k_{\alpha}} H_{1}(a) \\
C_{2 Q}(b) & =\int_{0}^{L} r^{2} H_{2}\left(k_{\beta} r\right) \mathrm{d} r \\
& =-\frac{3 L}{k_{\beta}^{2}} H_{0}(b)+\frac{3}{k_{\beta}^{2}} B_{1}(b)-\frac{L^{2}}{k_{\beta}} H_{1}(b) .
\end{aligned}
$$

The evaluation of $C_{1 Q}$ makes use of the recurrence relation for the Hankel function, Eq. (A3), to yield

$$
\begin{aligned}
C_{1 Q}= & \int_{0}^{L} r^{2} H_{2}\left(k_{\beta} r\right) \mathrm{d} r-\frac{1}{k_{\beta}} \int_{0}^{L} r H_{1}\left(k_{\beta} r\right) \mathrm{d} r \\
& -\frac{\xi}{k_{\beta}} \int_{0}^{L} r H_{1}\left(k_{\alpha} r\right) \mathrm{d} r .
\end{aligned}
$$

These integrals may be calculated as in Eq. (16),

$$
C_{1 Q}=C_{2 Q}(b)+\frac{L}{k_{\beta}^{2}}\left[H_{0}(b)+H_{0}(a)\right]-\frac{1}{k_{\beta}^{2}}\left[B_{1}(b)+B_{1}(a)\right] .
$$


3.4. Integration of $\int_{0}^{L} \frac{\partial G_{i j}}{\partial x_{k}} \mathrm{~d} r$

First, it must be observed that in this case, the tractions within the loaded element are anti-symmetric with respect to the loaded nodal point. Thus, the terms $H_{i j}^{l l}$ for $i, j=1,2$, can be calculated by integrating between the limits $e$ and $L_{1}$, (Fig. 3), because the integration along $[-e ; e]$ vanishes. Also, when the nodal point is at the centre of the element, the global integral vanishes.

Consider next the integration of $\left(\partial G_{i j} / \partial x_{k}\right)$ for $i, j, k=$ 1,2 . In view of Eq. (5), the integration of these terms may be written as

$\int_{e}^{L} \frac{\partial G_{i j}}{\partial x_{k}} \mathrm{~d} r=\int_{e}^{L} A_{x} \mathrm{~d} r+\int_{e}^{L} B_{x} \mathrm{~d}$

with

$A_{x} \frac{i}{4 \mu}\left[\delta_{i j}\left(C_{1}+C_{2}+C_{3}\right)-\left(\frac{\partial r}{\partial x_{i}}\right)\left(\frac{\partial r}{\partial x_{j}}\right) C_{4}\right] \frac{\partial r}{\partial x_{k}}$

$B_{x} \frac{i}{4 \mu}\left[2\left(\frac{\partial r}{\partial x_{i}}\right)\left(\frac{\partial r}{\partial x_{j}}\right) \frac{\partial r}{\partial x_{k}}-\left(\delta_{i k} \frac{\partial r}{\partial x_{j}}+\delta_{j k} \frac{\partial r}{\partial x_{i}}\right)\right] C_{5}$

$(i, j, k=1,2)$.

The first integral can easily be integrated by using the recurrence relations, Eqs. (A1)-(A4).

$\int_{e}^{L_{1}} A_{x} \mathrm{~d} r=\frac{i}{4 \mu}\left[C_{6}\right]_{e}^{L_{1}}$

with

$$
\begin{aligned}
C_{6}= & \delta_{i j}\left[-H_{0}\left(k_{\beta} r\right)+\frac{1}{k_{\beta} r}\left\{H_{1}\left(k_{\beta} r\right)-\xi H_{1}\left(k_{\alpha} r\right)\right\}\right] \\
& -\left(\frac{\partial r}{\partial x_{i}}\right)\left(\frac{\partial r}{\partial x_{j}}\right)\left\{H_{2}\left(k_{\beta} r\right)-\xi^{2} H_{2}\left(k_{\alpha} r\right)\right\} .
\end{aligned}
$$

For the integral $B_{4}=\int_{e}^{L_{1}} C_{5} \mathrm{~d} r$, it is necessary to use the recurrence relation for the Hankel functions Eq. (A1), which yields

$B_{4}=\frac{1}{2}\left[-H_{0}\left(k_{\beta} r\right)-H_{2}\left(k_{\beta} r\right)+\xi^{2}\left(H_{0}\left(k_{\alpha} r\right)+H_{2}\left(k_{\alpha} r\right)\right)\right]_{e}^{L}$.

3.5. Integration of $\int_{0}^{L} r \frac{\partial G_{i j}}{\partial x_{k}} \mathrm{~d} r$

In the light of Eq. 5, this integral may be written as

$\int_{0}^{L} r \frac{\partial G_{i j}}{\partial x_{k}} \mathrm{~d} r=\int_{0}^{L} r A_{x} \mathrm{~d} r+\int_{0}^{L} r B_{x} \mathrm{~d} r \quad i, j, k=1,2$

The first term can be evaluated making use of integration by parts

$\int_{0}^{L} r A_{x} \mathrm{~d} r=\operatorname{Lim}_{\varepsilon \rightarrow 0}\left[r C_{6}\right]_{\varepsilon}^{L}-\int_{0}^{L} C_{6} \mathrm{~d} r$.
To determine the lower limit of $\operatorname{Lim}_{\varepsilon \rightarrow 0}\left[r C_{6}\right]$ it is necessary to once again make use of the ascending series for the Bessel functions, Eqs. A5, and apply the known value for the limit $\operatorname{Lim}_{\varepsilon \rightarrow 0}\left\lfloor\left(k_{\beta} r\right) \ln \left(k_{\beta} r\right)\right\rfloor_{\varepsilon}=0$ and to observe that the terms attributable to the singularity vanish exactly on the account of the fact that $\xi=\beta / \alpha=k_{\alpha} / k_{\beta}$.

The integration of the term $\int_{0}^{L} C_{6} \mathrm{~d} r$ is obtained as in Eqs. (7), (9) and (10),

$\int_{0}^{L} C_{6} \mathrm{~d} r=\delta_{i j}\left(-B_{1}(b)+B_{2}\right)-\frac{\partial r}{\partial x_{i}} \frac{\partial r}{\partial x_{j}} B_{3}$.

As for the second integral, $\int_{0}^{L} r B_{x} \mathrm{~d} r$, requires the integration of $\int_{0}^{L} r C_{5} \mathrm{~d} r$, which was previously obtained in Eq. (10),

$$
\begin{aligned}
B_{3} & =\int_{0}^{L} r C_{5} \mathrm{~d} r=\int_{0}^{L}\left[H_{2}\left(k_{\beta} r\right)-\xi^{2} H_{2}\left(k_{\alpha} r\right)\right] \mathrm{d} r \\
& =B_{2}-\frac{1}{k_{\beta}}\left[H_{1}(b)-\xi H_{1}(a)\right] .
\end{aligned}
$$

3.6. Integration of $\int_{0}^{L} r^{2} \frac{\partial G_{i j}}{\partial x_{k}} \mathrm{~d} r$

In view of Eq. (5), this integral may be expressed as

$\int_{0}^{L} r^{2} \frac{\partial G_{i j}}{\partial x_{k}} \mathrm{~d} r=\int_{0}^{L} r^{2} A_{x} \mathrm{~d} r+\int_{0}^{L} r^{2} B_{x} \mathrm{~d} r \quad i, j, k=1,2$.

The first term can be integrated by parts,

$$
\int_{0}^{L} r^{2} A_{x} \mathrm{~d} r=\operatorname{Lim}_{\varepsilon \rightarrow 0}\left[r^{2} C_{6}\right]_{\varepsilon}^{L}-2 \int_{0}^{L} r C_{6} \mathrm{~d} r .
$$

The lower limit can yet again be shown to vanish by means of the ascending series for the Bessel functions, Eqs. (A5)(A8), and observing that $\operatorname{Lim}_{\varepsilon \rightarrow 0}\left\lfloor\left(k_{\beta} r\right)^{2} \ln \left(k_{\beta} r\right)\right\rfloor_{\varepsilon}=0$. The terms attributable to the singularity then gets cancelled because $\xi=\beta / \alpha=k_{\alpha} / k_{\beta}$.

The integration of $\int_{0}^{L} r C_{6} \mathrm{~d} r$ can be obtained as in Eq. (11),

$$
\begin{aligned}
\int_{0}^{L} r C_{6} \mathrm{~d} r= & \frac{i}{4 \mu} \int_{0}^{L}\left[\delta _ { i j } \left(-r H_{0}\left(k_{\beta} r\right)+\frac{r}{k_{\beta} r}\left\{H_{1}\left(k_{\beta} r\right)\right.\right.\right. \\
& \left.\left.-\xi H_{1}\left(k_{\alpha} r\right)\right\}\right)-\left(\frac{\partial r}{\partial x_{i}}\right)\left(\frac{\partial r}{\partial x_{j}}\right)\left\{r H_{2}\left(k_{\beta} r\right)\right. \\
& \left.\left.-\xi^{2} r H_{2}\left(k_{\alpha} r\right)\right\}\right] \mathrm{d} r \\
= & \frac{i}{4 \mu}\left[\delta_{i j}\left(-B_{1 L}(b)+B_{2 L}\right)-\left(\frac{\partial r}{\partial x_{i}}\right)\left(\frac{\partial r}{\partial x_{j}}\right) B_{3 L}\right] .
\end{aligned}
$$




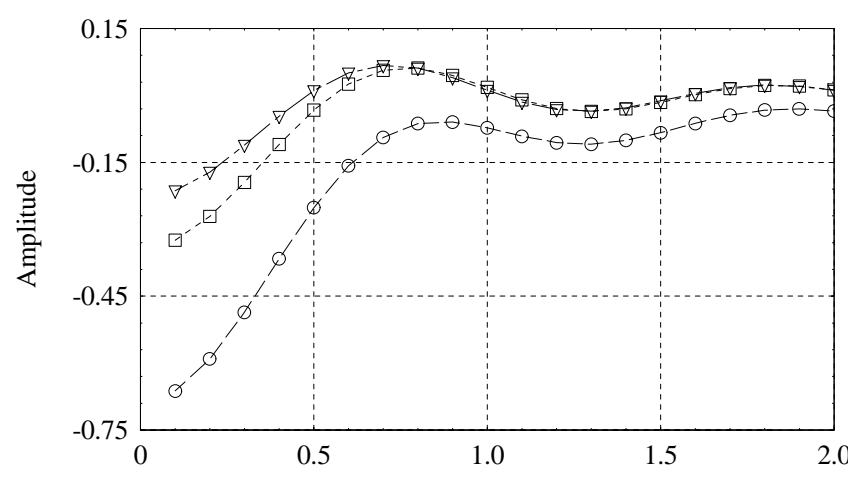

(a) $\int \phi G_{y y} d r$

$\nabla-\longrightarrow$ Quadratic

$\square---\boxminus$ Linear

๑-๑ Constant

$\frac{\omega L}{(2 \pi \beta)}$

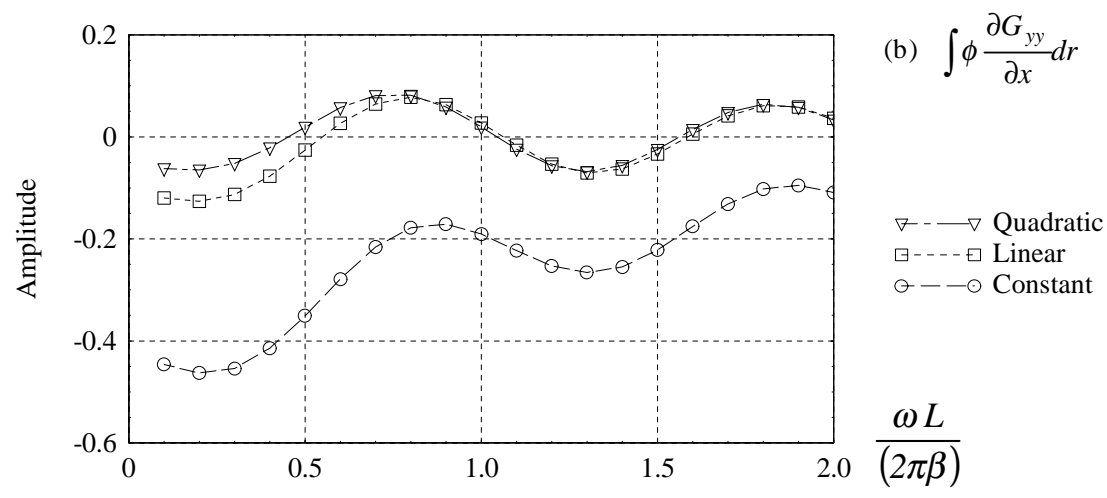

Fig. 4. The real part of the analytical integration of $\phi G_{y y}$ and $\phi \frac{\partial G_{y y}}{\partial x}\left(\phi=1, r, r^{2}\right)$.

The integration of $\int_{0}^{L} r^{2} A_{x} \mathrm{~d} r$ can thus be written as

$$
\begin{gathered}
\int_{0}^{L} r^{2} A_{x} \mathrm{~d} r=\frac{i}{4 \mu}\left[L ^ { 2 } \left(\delta_{i j}\left(-H_{0}(b)+\frac{1}{b}\left\{H_{1}(b)-\xi H_{1}(a)\right\}\right)\right.\right. \\
\left.-\left(\frac{\partial r}{\partial x_{i}}\right)\left(\frac{\partial r}{\partial x_{j}}\right)\left\{H_{2}(b)-\xi^{2} H_{2}(a)\right\}\right) \\
\left.-2\left(\delta_{i j}\left(-B_{1 L}(b)+B_{2 L}\right)-\left(\frac{\partial r}{\partial x_{i}}\right)\left(\frac{\partial r}{\partial x_{j}}\right) B_{3 L}\right)\right] .
\end{gathered}
$$

The second integral $\int_{0}^{L} r^{2} B_{x} \mathrm{~d} r$ reduces to the integration of $\int_{0}^{L} r^{2} C_{5} \mathrm{~d} r$, which can be performed as in Eq. (14),

$$
\int_{0}^{L} r^{2} C_{5} \mathrm{~d} r=\int_{0}^{L}\left[r H_{2}\left(k_{\beta} r\right)-\xi^{2} r H_{2}\left(k_{\alpha} r\right)\right] \mathrm{d} r=B_{3 L} .
$$

\section{Analytical versus numerical integration}

The analytical expressions previously derived are next compared with the results provided by the Gaussian quadrature using 2, 4 and 6 points. The singular integrals

$$
\begin{aligned}
& \int_{0}^{L} G_{i j} \mathrm{~d} r ; \quad \int_{0}^{L} r G_{i j} \mathrm{~d} r ; \quad \int_{0}^{L} r^{2} G_{i j} \mathrm{~d} r ; \quad \int_{e}^{L} \frac{\partial G_{i j}}{\partial x_{k}} \mathrm{~d} r ; \\
& \int_{0}^{L} r \frac{\partial G_{i j}}{\partial x_{k}} \mathrm{~d} r ; \quad \int_{0}^{L} r^{2} \frac{\partial G_{i j}}{\partial x_{k}} \mathrm{~d} r
\end{aligned}
$$

for $(i, j, k=1,2 e=0.02 \mathrm{~m})$,

are computed along a horizontal straight line ( $x$ direction) of length $L$. Computations are performed in the frequency range $(10-200 \mathrm{~Hz})$ at increments of $10 \mathrm{~Hz}$. The medium is characterized by a shear wave velocity of $100 \mathrm{~m} / \mathrm{s}$ and a compressional wave velocity of $200 \mathrm{~m} / \mathrm{s}$. Typical results are displayed in Figs. 4-6.

Fig. 4 depicts the exact values of the integrals for $\phi G_{y y}$ and $\phi\left(\partial G_{y y}\right) /(\partial x)\left(\phi=1, r, r^{2}\right)$, while Figs. 5 and 6 illustrate the error committed by using the numerical integration. As can be seen, the agreement between the numerical and analytical values improves with the number of Gaussian stations, and the results are virtually exact when using six points. On the other hand, these comparisons indicate that two Gaussian points are generally not enough for accurate results. Extensive additional numerical tests for the other integrals confirmed the validity of the analytical expressions presented here. 

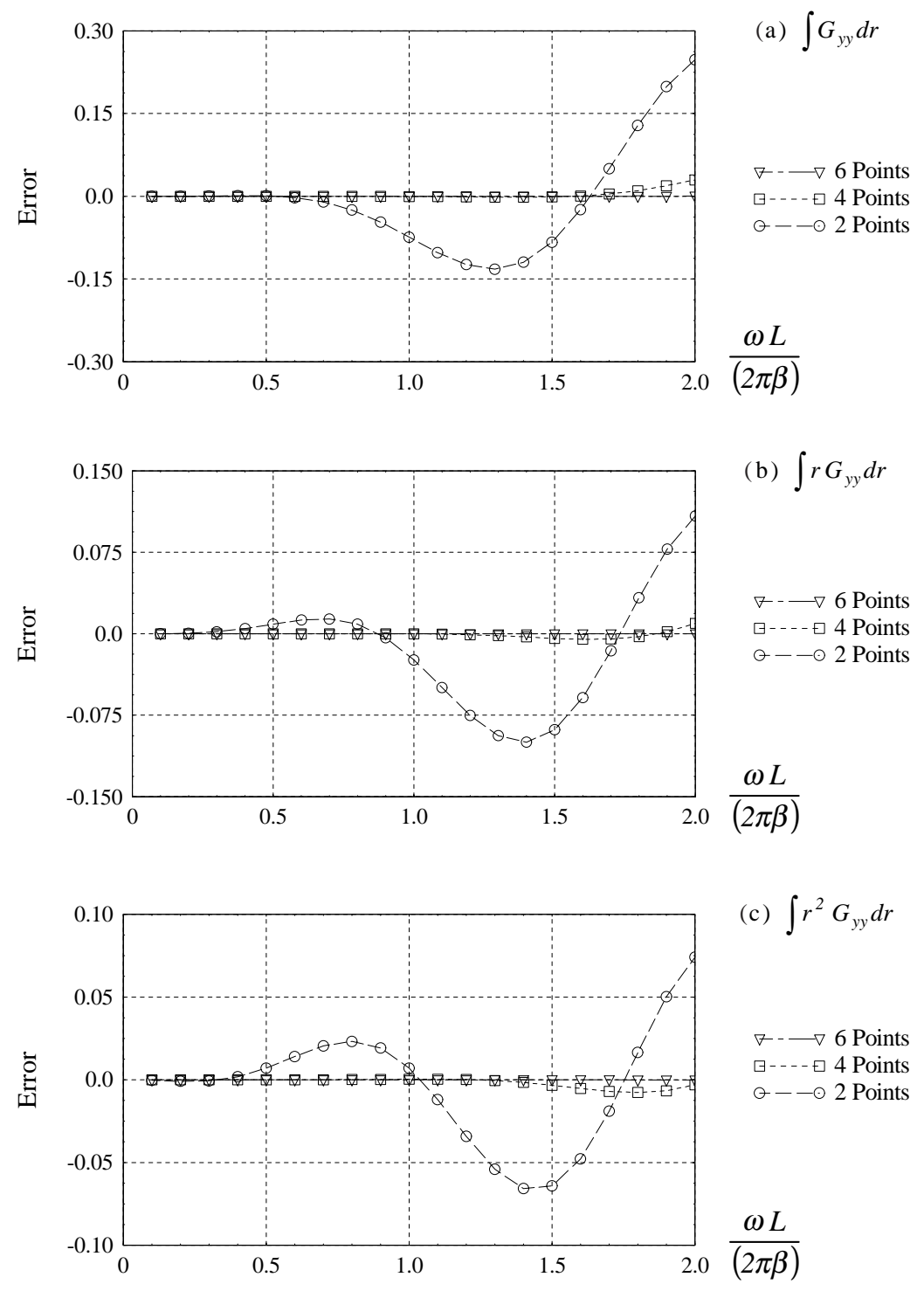

Fig. 5. The real part of the analytical integration of $\phi G_{y y}\left(\phi=1, r, r^{2}\right)$.

\section{Example of application}

The equations developed previously were implemented in a computer program and used to carry out simulations for problems of wave scattering. To check the correctness of the program, the elastic inclusion was first assigned the same material properties as the external medium, which indeed reproduced the free field conditions. Simulations were then carried out for both cylindrical cavity and elastic inclusion in the path of a dilatational wave field. In as much as the results of the cavity and the elastic inclusion are qualitatively similar, only the latter will be presented.

Consider an elastic inclusion in an unbounded homogeneous medium, as shown in Fig. 7. A compressive source is applied at point $O$, out of which only $P$ waves emanate. In the absence of the inclusion, this source elicits particle motions along radial directions only, whose amplitude is $u_{r}^{\text {inc }}=-k_{\alpha 1} H_{1}\left(k_{\alpha 1} r\right)$, where $k_{\alpha 1}=\omega / \alpha_{1}$ is the wavenumber, $\omega$ the circular frequency, $\alpha_{1}$ the velocity of dilatational waves in the medium surrounding the inclusion and $r$ the source-receiver distance. Upon impinging the inclusion, the incident $P$ waves reflect, refract and convert partially into other types of waves, which are then scattered in all directions.

The motions shown at the receiver is computed using constant, linear and quadratic boundary elements. Because of symmetry, the receiver does not move in the horizontal direction. The motion is also computed with the exact solution reported by Pao \& Mow [7].

Figs. 8 and 9 display, as a function of frequency, the real and imaginary parts as well as the amplitude of the vertical displacement associated with the scattered field. The results are computed at 64 frequencies in the range 1-64 Hz. This corresponds to incident $P$ waves with wavelengths between 

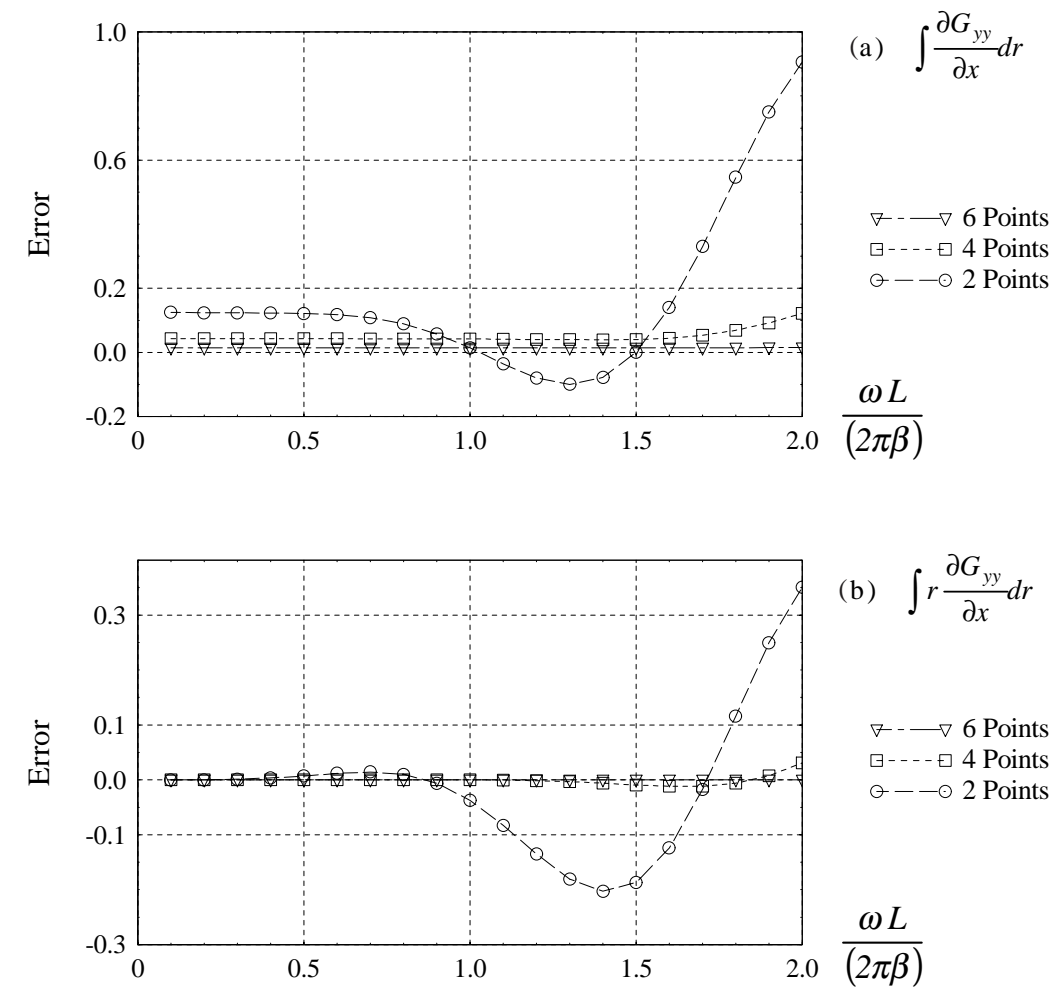

$$
\begin{aligned}
& \text { (b) } \int r \frac{\partial G_{y y}}{\partial x} d r \\
& \nabla-\longrightarrow 6 \text { Points } \\
& \square---\square \text { Points } \\
& \text { ๑- }-\odot 2 \text { Points }
\end{aligned}
$$

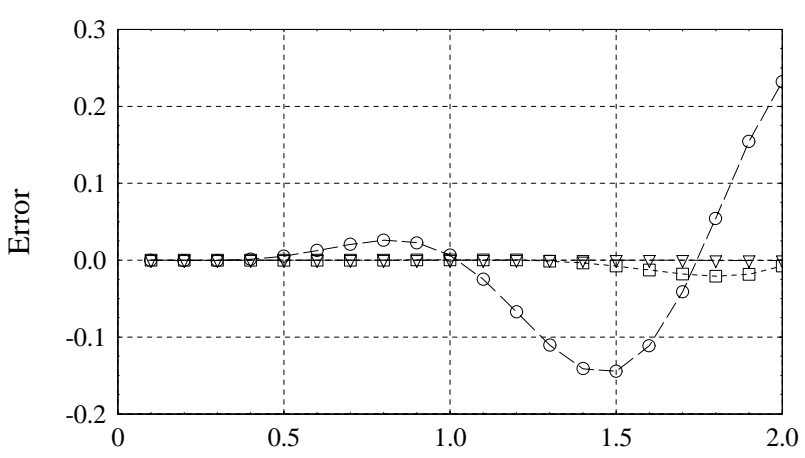

(c) $\int r^{2} \frac{\partial G_{y y}}{\partial x} d r$

\section{$\frac{\omega L}{(2 \pi \beta)}$}

Fig. 6. The real part of the analytical integration of $\phi \frac{\partial G_{y y}}{\partial x}\left(\phi=1, r, r^{2}\right)$.

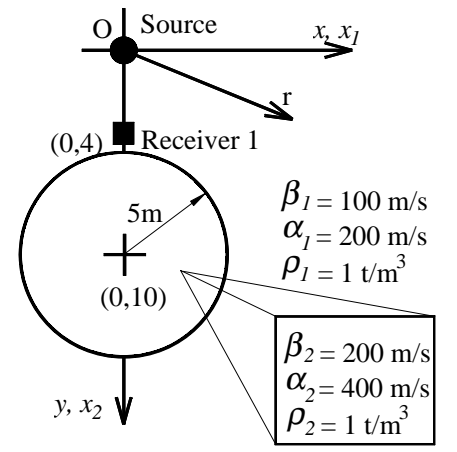

Fig. 7. The cylindrical inclusion in an unbounded medium.
20.0 and 0.3125 times the diameter of the cylinder. The inclusion is modelled with 25 and 50 boundary elements, and results are shown in Figs. 8 and 9, respectively. Not surprisingly, the results improve both with the number and order of the boundary elements.

Figs. 10 and 11 display the same information as Figs. 8 and 9, but expressed in terms of the number of elements, and for a pulsating source vibrating at a single frequency, namely 32 and $64 \mathrm{~Hz}$. These two frequencies correspond to incident waves with wavelengths of 6.25 and $3.125 \mathrm{~m}$, respectively. The results confirm yet again that the response improves with the expansion order and number of elements used to model the cavity, as expected. More importantly, these results provide confirmation of the correctness of the analytical integrals presented here. 


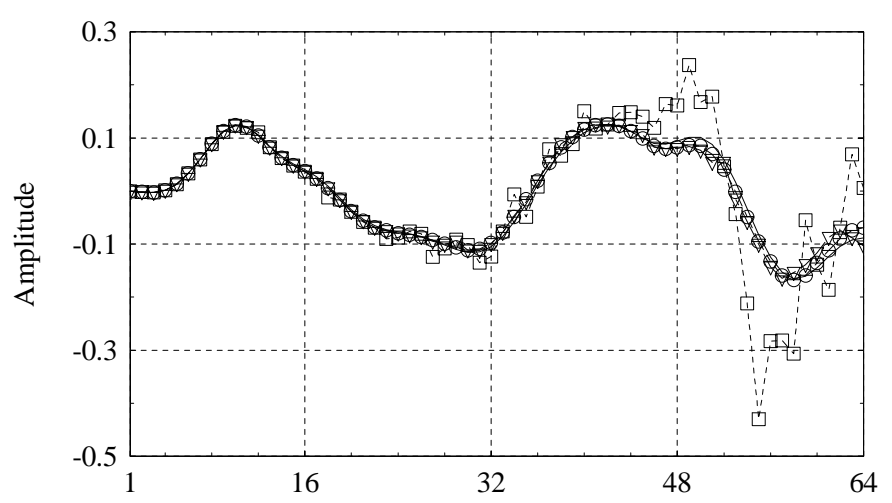

(a) Real part

$$
\begin{aligned}
& \ominus \cdots-\ominus \text { Quadratic } \\
& \nabla-\square-\nabla \text { Linear } \\
& \square-\cdots \text { Constant } \\
& \square \text { Analytical }
\end{aligned}
$$

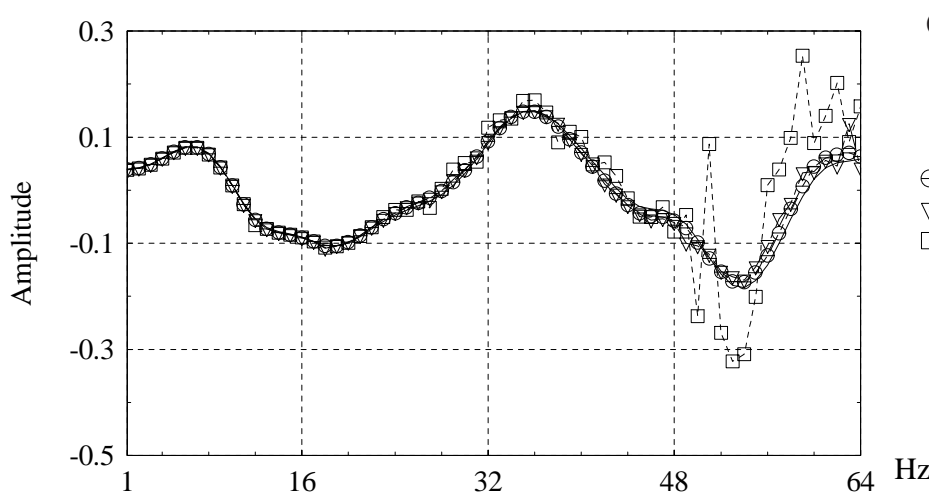

(b) Imaginary part

$$
\begin{aligned}
& \ominus--\ominus \text { Quadratic } \\
& \nabla-\square-\nabla \text { Linear } \\
& \square-\cdots \text { Constant } \\
& \square \text { Analytical }
\end{aligned}
$$

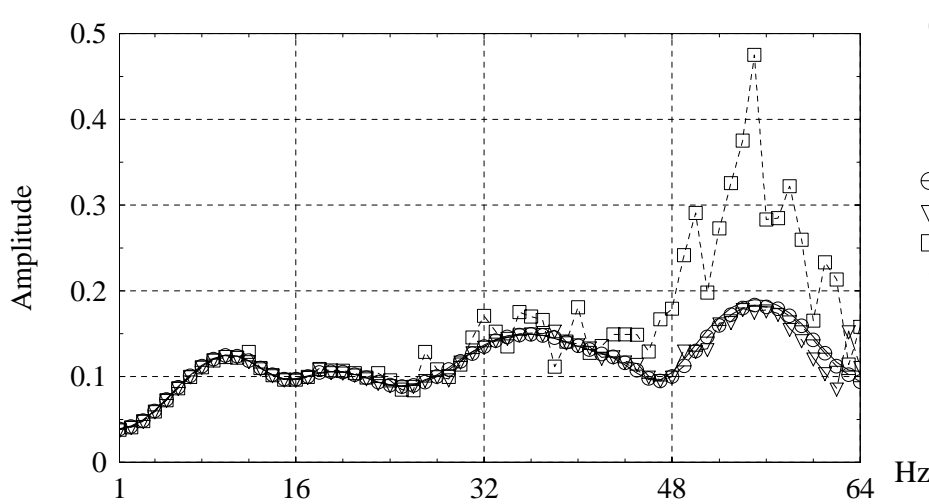

(c) Modulus

$$
\begin{aligned}
& \ominus \cdots \ominus \text { Quadratic } \\
& \nabla-\square \text { Linear } \\
& \square-\cdots \text { Constant } \\
& \square \text { Analytical }
\end{aligned}
$$

Fig. 8. The in-plane vertical field scattered by a cylindrical elastic inclusion modelled with 25 boundary elements.

\section{Conclusions}

This paper presented exact expressions for the singular integrals involved in the application of the BEM to problems of wave motion in plane strain (SV-P case). Boundary elements with constant, linear and quadratic interpolation functions were considered and implemented in a computer program. The correctness of these expressions was then tested by means of an ideal problem for which the exact solution is known, namely the problem of waves diffracted and scattered by a cylindrical inclusion embedded in homogeneous medium when illuminated by a $P$ line source.

Comparisons of the analytical integrals presented with those obtained by numerical quadrature demonstrated on the one hand the correctness of the theoretical formulae. 

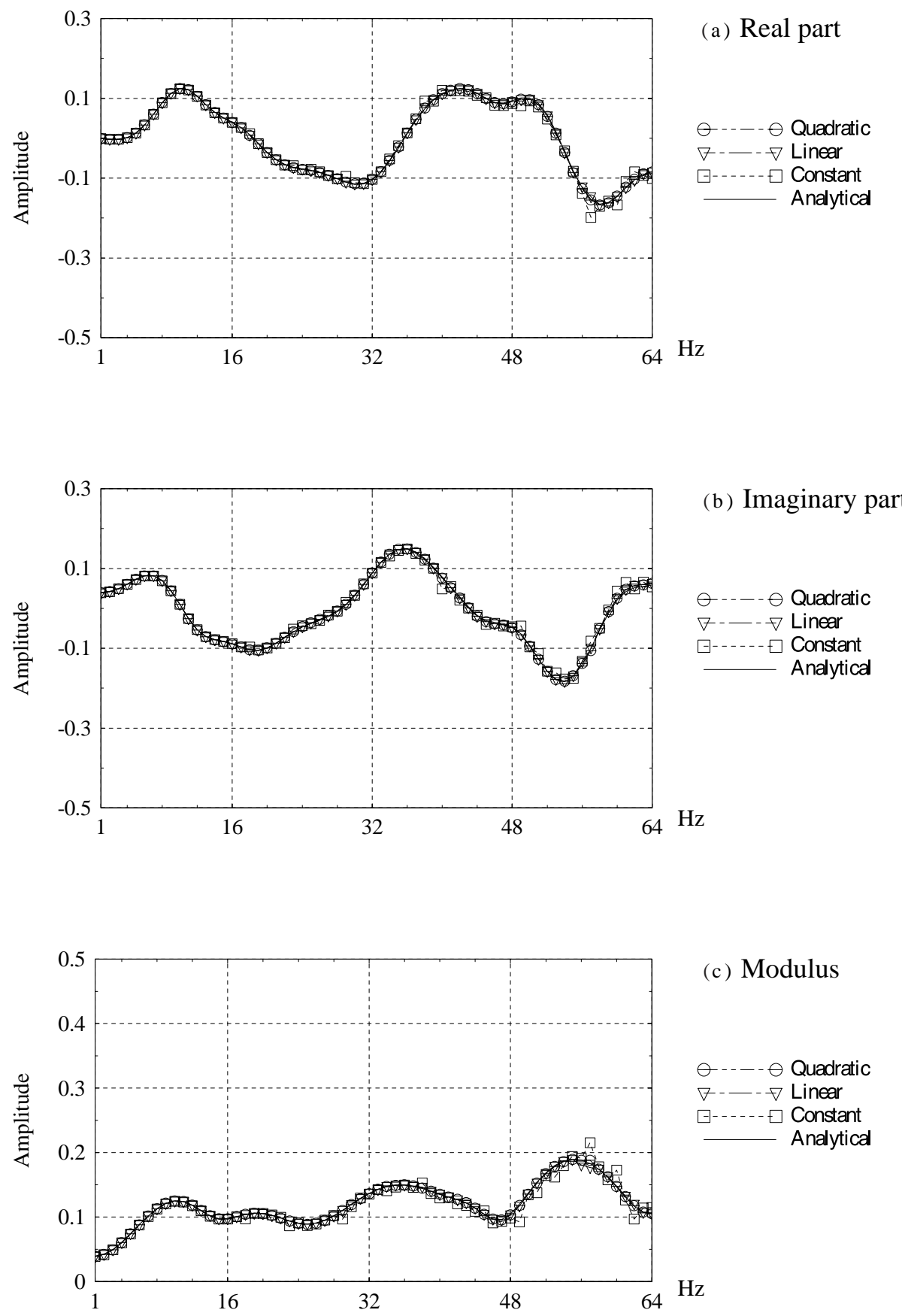

Fig. 9. The in-plane vertical field scattered by a cylindrical elastic inclusion modelled with 50 boundary elements.

On the other hand, it was observed that accurate numerical integration via Gauss-Legendre quadrature requires at least four, if not six points for reliably accurate results.

The results obtained with the BEM formulation for the waves scattered by an elastic inclusion were in excellent agreement with the known analytical solution for this problem. As expected, the agreement improved with both the expansion order and grid refinement (i.e. size of the elements). From a practical point of view, however, the improvements in accuracy and efficiency that can be obtained by using higher order elements is offset by the increased demands that they place on a $C P U$ time. As is well known, however, higher order elements may be indispensable in regions where large strain gradients can be expected.

All of the previous findings provided a firm validation for the correctness of the singular integrals presented here. 

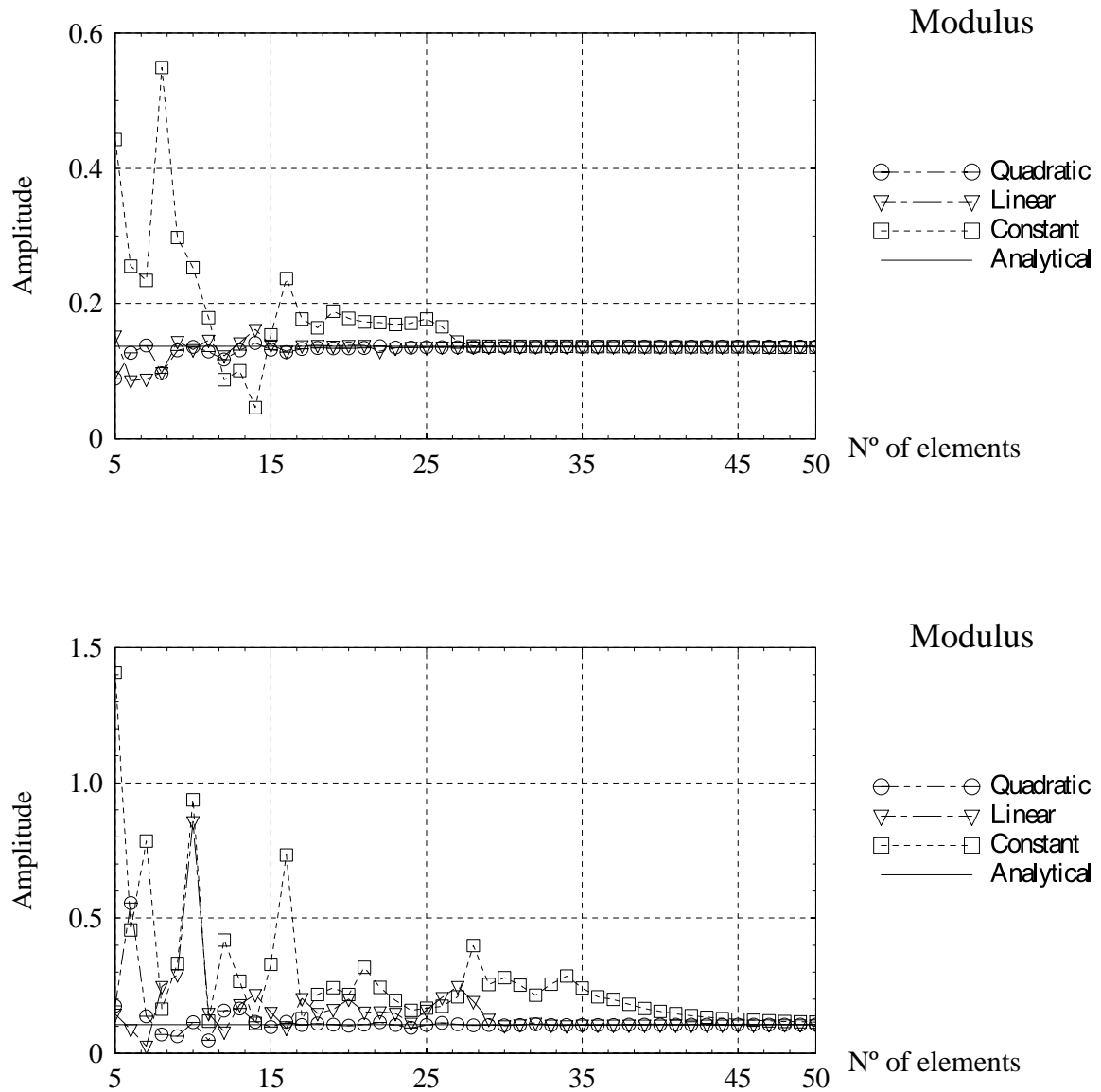

Fig. 11. The in-plane vertical field scattered by a cylindrical elastic inclusion subjected to a $64 \mathrm{~Hz}$ source.

\section{Appendix A}

\section{A.1. Recurrence relations for the Bessel functions}

$\frac{\mathrm{d} \vartheta_{\nu}(k r)}{\mathrm{d} r}=-\frac{\nu \vartheta_{\nu}(k r)}{r}+k \vartheta_{\nu-1}(k r)$

$\frac{\mathrm{d} \vartheta_{\nu}(k r)}{\mathrm{d} r}=\frac{\nu \vartheta_{\nu}(k r)}{r}-k \vartheta_{\nu+1}(k r)$

$\frac{2 \nu}{k r} \vartheta_{\nu}(k r)-\vartheta_{\nu-1}(k r)=\vartheta_{\nu+1}(k r)$

$r^{2} \vartheta_{\nu+1}(k r)=\frac{r}{k} \vartheta_{\nu}(k r)-\frac{r^{2}}{k} \frac{\mathrm{d} \vartheta_{\nu}(k r)}{\mathrm{d} r}$

$\vartheta$ denotes $J, Y, H$, or any other linear combination of these functions.

\section{A.2. Ascending series for the Bessel functions}

$J_{\nu}(z)=\left(\frac{1}{2} z\right)^{\nu} \sum_{k=0}^{\infty} \frac{\left(-\frac{1}{4} z^{2}\right)^{k}}{k ! \Gamma(\nu+k+1)}$

$$
\begin{gathered}
Y_{n}(z)=-\frac{\left(\frac{1}{2} z\right)^{-n}}{\pi} \sum_{k=0}^{n-1} \frac{(n-k-1) !}{k !}\left(\frac{1}{4} z^{2}\right)^{k}+\frac{2}{\pi} \ln \left(\frac{1}{2} z\right) J_{n}(z) \\
-\frac{\left(\frac{1}{2} z\right)^{n}}{\pi} \sum_{k=0}^{\infty}\{\psi(k+1)+\psi(n+k+1)\} \frac{\left(-\frac{1}{4} z^{2}\right)^{k}}{k !(n+k) !}
\end{gathered}
$$

where $\psi(n)$ is the Psi (Digamma) function.

$$
J_{0}(z)=1-\frac{\frac{1}{4} z^{2}}{(1 !)^{2}}+\frac{\left(\frac{1}{4} z^{2}\right)^{2}}{(2 !)^{2}}-\frac{\left(\frac{1}{4} z^{2}\right)^{3}}{(3 !)^{2}}+\cdots
$$

$$
\begin{aligned}
Y_{0}(z)= & \frac{2}{\pi}\left\{\ln \left(\frac{1}{2} z\right)+\gamma\right\} J_{0}(z) \\
& +\frac{2}{\pi}\left\{\frac{\frac{1}{4} z^{2}}{(1 !)^{2}}-\left(1+\frac{1}{2}\right) \frac{\left(\frac{1}{4} z^{2}\right)^{2}}{(2 !)^{2}}\right. \\
& \left.+\left(1+\frac{1}{2}+\frac{1}{3}\right) \frac{\left(\frac{1}{4} z^{2}\right)^{3}}{(3 !)^{2}}-\cdots\right\} .
\end{aligned}
$$




\section{References}

[1] Tadeu AJB, Santos PFA, Kausel E. Closed-form integration of singular terms for constant, linear and quadratic boundary elements. Part I. SH wave propagation, Engineering Analysis with Boundary Elements 1999;23(8):671-81.

[2] Tadeu AJB, Kausel E, Vrettos C. Modelling and seismic imaging of buried structures. Soil Dyn Earthquake Engng 1996;15:387-97.

[3] Tadeu AJB. Modelling and seismic imaging of buried structures, $\mathrm{PhD}$ thesis, Department of Civil Engineering, M.I.T., Cambridge, MA, 1992.
[4] Manolis GD, Beskos DE. Boundary element methods in elastodynamics, London: Unwin Hyman, 1988 sold to Chapman and Hall.

[5] Dominguez J, Abascal R. On fundamental solutions for the boundary integral equations method in static and dynamic elasticity. Engng Analysis 1984;1:128-34.

[6] Abramowitz M, Stegun IA. Handbook of mathematical functions, New York: Dover, 1964. p. 480-4.

[7] Pao YH, Mow CC. Diffraction of elastic waves and dynamic stress concentrations, Crane and Russak, 1973. 